\title{
ESTUDO GRANULOMÉTRICO DE CHOCOLATES ARTESANAIS ELABORADOS EM DIFERENTES MOINHOS
}

\author{
L. A. QUEMELLI ${ }^{1 *}$, B. P. NASCIMENTO ${ }^{1}$, L. S. ARRIECHE ${ }^{2}$ \\ ${ }^{1}$ Alunos de Iniciação Científica, Universidade Federal do Espírito Santo (CEUNES) \\ ${ }^{2}$ Professor, Universidade Federal da Integração Latino Americana, Instituto Latino \\ Americano de Tecnologia, Infraestrutura e Território \\ *E-mail para contato: lucas_lq8@hotmail.com
}

\begin{abstract}
RESUMO - O diâmetro das partículas do chocolate é uma propriedade que deve ser inferior a $20 \mu \mathrm{m}$. Assim, investigou-se a granulometria de um chocolate artesanal elaborado em moinho de rolos cônicos e em moinho de bolas. Amostras dispersas em óleo mineral foram colocadas sobre câmara de Neubauer, com escala de $250 \mu \mathrm{m}$. Imagens foram capturadas em um microscópio óptico, com aumento de 40 vezes, para a determinação de diâmetro e forma. Modelos clássicos como o GGS, RRB e log-normal foram ajustados aos dados. Os perfis granulométricos foram estatisticamente bem representados pelo modelo $\mathrm{RRB}$, com $\mathrm{R}^{2}$ superior a 0,99, a um nível de significância de 5\%. O moinho de rolos demandou menor tempo para reduzir as partículas à especificação e proporcionou melhor fluidez.
\end{abstract}

\section{INTRODUÇÃ̃O}

O chocolate originou-se no povo Asteca, que o consumia na forma de bebida. Era elaborado com as amêndoas da árvore Theobroma cacao. Posteriormente, foi processado com adição de açúcar pelos europeus (SCHUMACHER, 2008). Um estudo de Schmitz (2001), sinaliza seus efeitos benéficos à saúde. Segundo Oetterer et. al., (2006), o processamento engloba a moagem, em que as partículas são reduzidas de tamanho. $\mathrm{O}$ concheamento confere textura característica e reduz a acidez. No refino, reduz-se os cristais para em torno de $20 \mu \mathrm{m}$.

Para obtenção das distribuições de tamanho das partículas de chocolate, existem três modelos clássicos: o de Gates, Gaudin e Schumann (GGS), o de Rosin, Rammler e Bennet (RRB), e o modelo que estabelece a função $X=X$ (D) no formato log-normal (MASSARANI, 1984). A partir desses modelos é possível obter parâmetros de importante significado físico. Assim, elaborou-se um chocolate artesanal em moinhos de rolos e de bolas, a fim de comparar esses equipamentos para a produção do chocolate, em função da distribuição dos diâmetros de partícula do produto e o tempo de processamento.

\section{METODOLOGIA}

\subsection{Materiais e equipamentos}

O chocolate foi elaborado com dois ingredientes: nibs de cacau e açúcar cristal. 
Utilizou-se o moinho de rolos cônicos fixos, com rotação de $1100 \mathrm{rpm}$, e dimensões do moinho de $46(\mathrm{~cm}) \times 28(\mathrm{~cm}) \times 28(\mathrm{~cm})$; moinho de bolas com 45 bolas, sendo 30 bolas com diâmetros de $25 \mathrm{~mm}$ e 15 bolas de $30 \mathrm{~mm}$; balança de precisão analítica; câmera de Neubauer; microscópio óptico; micrômetro digital; termômetro infravermelho; termopar; secador de convecção forçada portátil, com aquecimento por resistências elétricas, além de vidrarias de laboratório.

\subsection{Métodos}

Elaboração do chocolate e análise do material particulado: Para os experimentos no moinho de rolos foram elaborados $500 \mathrm{~g}$ de chocolate, com $70 \%$ em massa de cacau, em seis horas. Já no moinho de bolas, foram realizados experimentos variando o percentual de cacau entre 70 e $100 \%$, com e sem aquecimento, promovido pelo secador portátil, conforme Tabela 1. Nesse moinho, utilizou-se massa de 150 g. Durante a elaboração dos chocolates, amostras foram coletadas a cada 30 minutos, para análise das partículas de diâmetro máximo, em um micrômetro digital. Para análise no microscópio, foram preparadas quatro dispersões, com as amostras finais de chocolate produzido, tendo como dispersante o óleo mineral. Posteriormente, cada amostra foi colocada sobre câmara de Neubauer, para serem fotografadas em um microscópio óptico, com aumento de quarenta vezes.

Tabela 1 - Planejamento de experimento.

\begin{tabular}{|c|c|c|}
\hline Experimento & Composição & Aquecimento \\
\hline 1 & Nibs & Não \\
\hline 2 & Nibs & Sim \\
\hline 3 & $70 \%$ Nibs $+30 \%$ Açúcar & Sim \\
\hline 4 & $70 \%$ Nibs $+30 \%$ Açúcar & Sim \\
\hline
\end{tabular}

\subsection{Cálculo do diâmetro médio de Sauter}

A obtenção representativa do diâmetro médio de partículas de uma amostra pode ser determinada por meio da Equação 1, que representa o diâmetro médio de Sauter, caracterizando-se como um dos métodos mais utilizados em sistemas particulados, sendo que o $x_{\mathrm{i}}$ representa a distribuição de frequência e o $D_{i}$ representa o diâmetro médio entre fração mássica passante e a retida (CREMASCO, 2012).

$$
d p_{s}=1 /\left(\sum_{i=1}^{n} x_{i} / D_{i}\right)
$$

O diâmetro de Sauter é uma medida mais precisa quando comparada à média ponderada, utilizada para se encontrar o diâmetro médio da amostra. A precisão associada ao diâmetro médio de Sauter está na utilização dos parâmetros que caracterizam a amostra.

\subsection{Modelos de distribuição granulométrica}

O diâmetro de Sauter pode ser obtido também por meio dos modelos clássicos de distribuição granulométrica: Gates, Gaudin e Schumann (GGS), Rosin, Rammler e Bennet (RRB) e log-normal (MASSARANI, 1984), conforme Tabela 2, 
Tabela 2 - Modelos de distribuição granulométrica.

\begin{tabular}{|c|c|l|l|}
\hline Modelo & Parâmetros & \multicolumn{1}{|c|}{ Equação característica } & \\
\hline GGS & $\mathrm{k}, \mathrm{m}$ & $X_{i}=\left(D_{i} / k\right)^{m}$ & (2) \\
\hline RRB & $\mathrm{D}^{\prime}, \mathrm{n}$ & $X_{i}=1-\exp \left[-\left(D_{i} / D^{\prime}\right)^{n}\right]$ & \\
& & $X_{i}=1 / 2\left[1+\operatorname{erf}\left(Z_{i}\right)\right]$ & \\
\hline log-normal & $\sigma, \mathrm{D}$ & $(4)$ \\
\hline
\end{tabular}

em que:

$$
Z_{i}=\frac{\ln \left(D_{i} / D_{50}\right)}{\sqrt{2}(\ln \sigma)}
$$

A partir desses modelos e de seus parâmetros associados é possível estabelecer equações para o cálculo do diâmetro médio de Sauter, correspondente a cada modelo (CRESMASCO, 2012). Assim, ao conhecer o modelo de distribuição granulométrica que melhor se ajusta ao chocolate, o diâmetro médio de Sauter pode ser calculado a partir das expressões apresentadas na Tabela 3 (FRARE et al., 2000).

Tabela 3 - Diâmetro médio de Sauter.

\begin{tabular}{|c|c|c|}
\hline Modelo & $\mathrm{D}_{\mathrm{i}}$ & \\
\hline GGS & $d_{p s}=k\left(\frac{m-1}{m}\right), \mathrm{m}>1$ & \\
\hline RRB & $d_{p s}=\frac{D^{\prime}}{\Gamma(1-1 / n)}, \mathrm{n}>1$ & \\
\hline Log-normal & $d_{p s}=D_{50} \exp \left(-\frac{1}{2} \ln ^{2} \sigma\right)$ & \\
\hline
\end{tabular}

O ajuste dos modelos aos dados foi realizado por meio do método dos mínimos quadrados, a 95\% de confiança. Parâmetros estatísticos como o teste t, o erro relativo e o coeficiente de determinação foram utilizados para avaliação dos ajustes.

\section{RESULTADOS E DISCUSSÃO}

Os valores dos diâmetros máximos das partículas do chocolate medidos pelo micrômetro digital, após o processo ser realizado no moinho de bolas, estão apresentados na Figura 1. Observa-se que a diminuição do diâmetro das partículas do chocolate levou menor tempo quando houve aquecimento (Experimento 2) do que sem aquecimento (Experimento 1). Com a adição de açúcar cristal (Experimento 3), houve aumento no tempo. 


\section{Congresso Brasileiro de Engenharia Química \\ em Iniciação Científica \\ UFSCar - São Carlos - SP \\ 16 a 19 de julho de 2017}

Figura 1 - Diâmetro (mm) em função do tempo (min) para os experimentos 1, 2, 3 e 4.
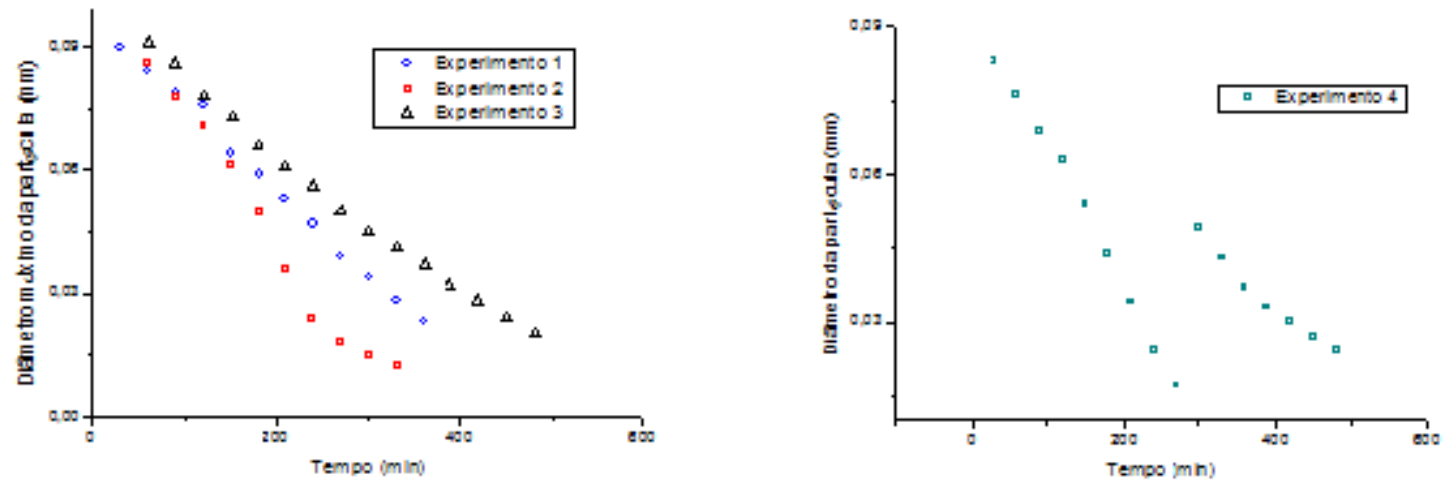

A descontinuidade na Figura 1 (experimento 4) corresponde à adição de açúcar cristal, próximo ao tempo de $300 \mathrm{~min}$. A temperatura do processo, no moinho de bolas, para todos os experimentos com aquecimento ficou entre 30 e $35{ }^{\circ} \mathrm{C}$. No processo sem aquecimento, a temperatura não ultrapassou $30{ }^{\circ} \mathrm{C}$. As imagens capturadas no microscópio óptico foram processadas e analisadas no software Image $J^{\circledR}$. Obteve-se as áreas projetadas de partículas para a determinação do diâmetro equivalente. Assim, foi possível elaborar os gráficos do diâmetro em função da fração cumulativa. Na Figura 2, apresenta-se dois resultados típicos.

Figura 2 - Distribuição granulométrica cumulativa com ajuste ao modelo RRB.
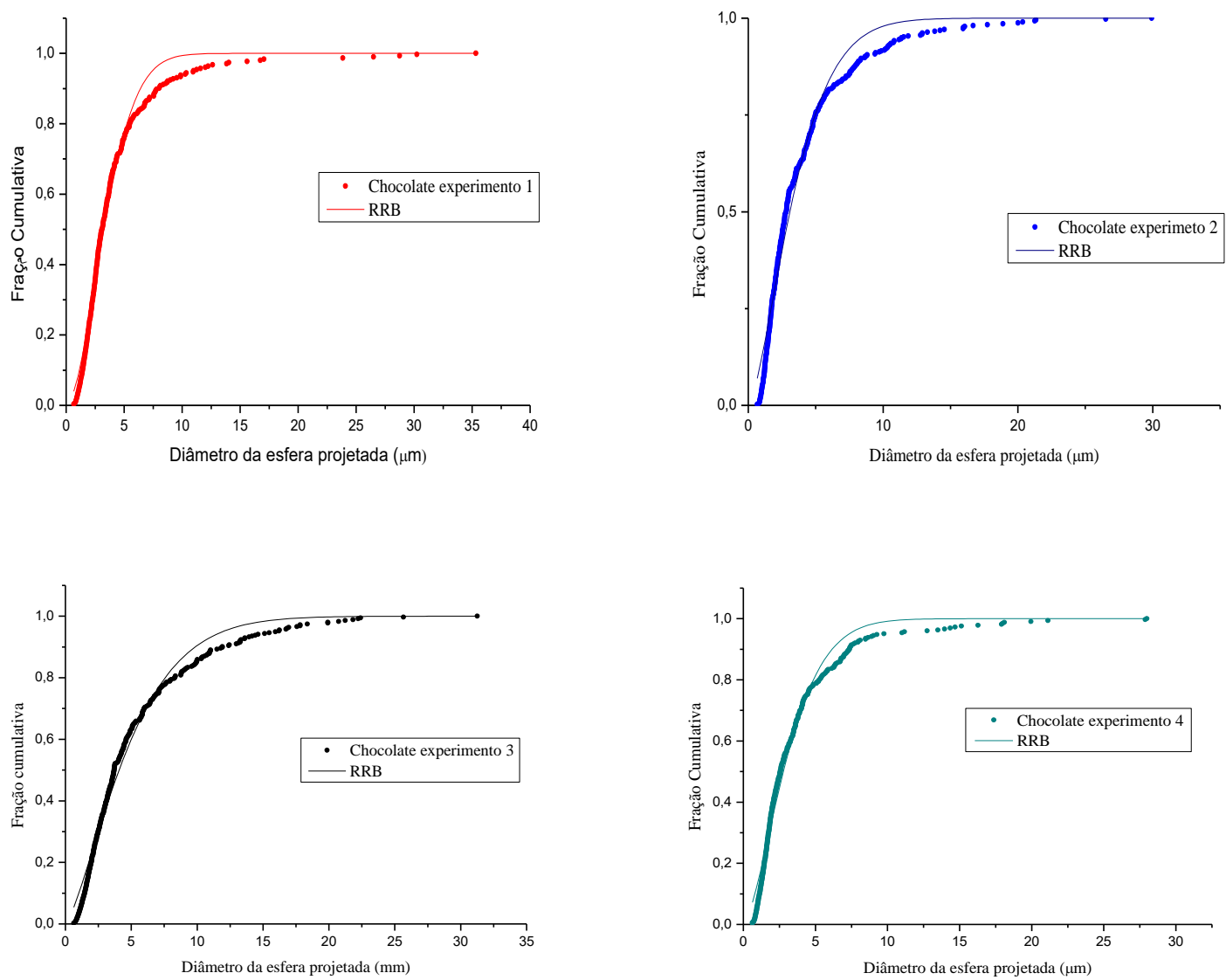
O modelo RRB teve maiores valores do coeficiente de determinação ( $R^{2}$ maior que 0,98) e menor erro relativo (em torno de $10 \%$ ). Na Tabela 3, apresenta-se um resultado típico. Foi o modelo que melhor se ajustou à distribuição das partículas do chocolate. Por isso, optou-se unicamente pela representação do ajuste ao modelo RRB na Figura 2, como visto anteriormente.

Tabela 4 - Modelos de distribuição granulométrica e seus parâmetros para o chocolate.

\begin{tabular}{|c|c|c|c|c|c|}
\hline Experimento & Modelo & $\mathrm{R}^{2}$ & Parâmetros & Teste $\mathrm{t}$ & Erro Relativo \\
\hline \multirow{6}{*}{1} & \multirow[t]{2}{*}{ GGS } & \multirow[t]{2}{*}{0,730} & $\mathrm{~m}=0,523$ & 28,555 & \multirow[t]{2}{*}{$28,72 \%$} \\
\hline & & & $\mathrm{k}=13,298$ & 24,856 & \\
\hline & \multirow[t]{2}{*}{ RRB } & \multirow[t]{2}{*}{0,985} & $\mathrm{n}=1,782$ & 35,779 & \multirow[t]{2}{*}{$10,96 \%$} \\
\hline & & & $\mathrm{D}^{\prime}=4,007 \mu \mathrm{m}$ & 206,907 & \\
\hline & \multirow[t]{2}{*}{ log-normal } & \multirow[t]{2}{*}{0,781} & $\mathrm{D}_{50}=1014,404 \mu \mathrm{m}$ & 6,038 & \multirow[t]{2}{*}{$800,63 \%$} \\
\hline & & & $\sigma=31.601$ & 32,217 & \\
\hline
\end{tabular}

Para cálculo dos diâmetros médios de Sauter utilizando a equação 7, para o modelo RRB, obteve-se um diâmetro de Sauter ao final do processo para os experimentos 1, 2, 3 e 4 de $1,991 \mu \mathrm{m}, 1,428 \mu \mathrm{m}, 1,627 \mu \mathrm{m}$ e $1,366 \mu \mathrm{m}$, respectivamente. E o diâmetro médio de Sauter ao final do processo, calculado a partir da equação 1, para os experimentos 1, 2, 3 e 4 foi de, respectivamente, 2,662 $\mu \mathrm{m}, 2,427 \mu \mathrm{m}, 2,196 \mu \mathrm{m}$ e 2,983 $\mu \mathrm{m}$. Também foram realizados experimentos no moinho de rolos, a fim de produzir um chocolate com $70 \% \mathrm{em}$ massa de cacau e $30 \%$ de açúcar cristal. O açúcar cristal foi adicionado próximo ao tempo de 360 min, conforme Figura 4.

Figura 4 - Diâmetro do chocolate em função do tempo para um processo de 480 min método de medição: microscopia óptica.

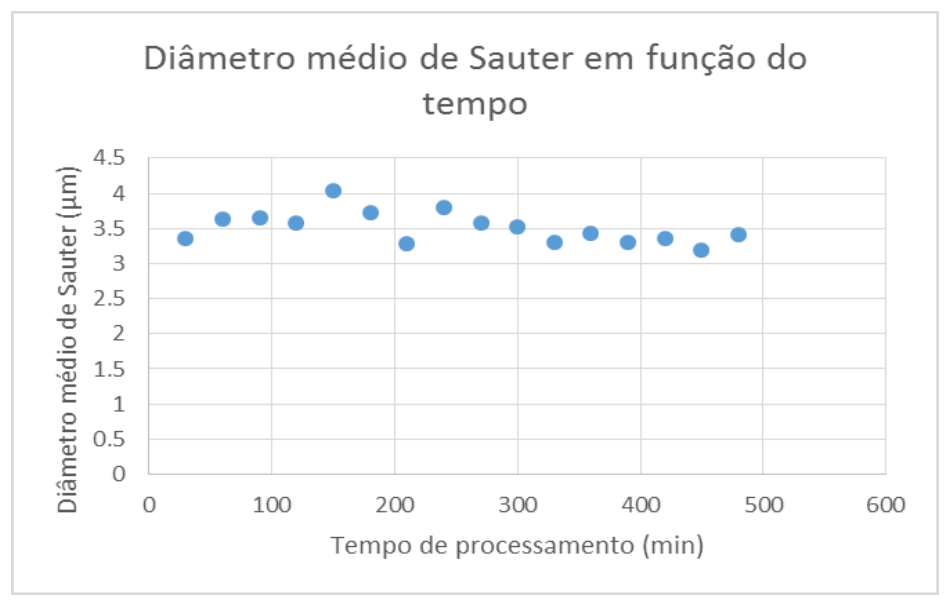

As amostras foram retiradas a cada trinta minutos. O valor de diâmetro muito pequeno, logo no início, é devido ao método de medição das partículas. Este considera a média dos valores de partículas de uma amostra, para então ser calculado outro valor médio: o diâmetro médio de Sauter. Ainda assim, podem ser notadas variações nos valores dos diâmetros médios de Sauter na Figura 4. Porém essas variações diminuem. Portanto, a tendência é se tornar constante. 


\section{CONCLUSÃO}

Para o chocolate produzido em moinho de bolas, obteve-se um melhor resultado quando foi utilizado aquecimento durante o processo, tanto na textura quanto no tempo de redução das partículas. Entretanto, foi visto que a adição de açúcar fez com que o processo ocorresse mais lentamente. Fica mais evidente no Experimento 4, quando há uma descontinuidade bem perceptível no gráfico. Também é observado que o modelo RRB foi o que teve melhor ajuste na distribuição granulométrica da análise final de cada chocolate produzido. $O$ modelo é valido na faixa de $0,6 \mu \mathrm{m}<\mathrm{D}_{\mathrm{i}}<40 \mu \mathrm{m}$ para o chocolate.

Foi evidenciado que as partículas de cacau no moinho de rolos têm um limite para ser reduzidas, que é uma região do gráfico entre 3 e $3,5 \mu \mathrm{m}$. O processo no moinho de rolos ocorre mais rapidamente. Além de o produto final possuir menor arenosidade, também foram observados menores valores de diâmetro médio de Sauter. O moinho de rolos permite que o chocolate possua aspecto mais próximo ao de fluido de Bingham - fluido não Newtoniano que descreve o comportamento do chocolate - sem utilizar fonte externa de aquecimento no sistema. Dessa forma, essas informações são úteis na seleção da melhor tecnologia e na busca das condições ótimas de processo de modo que as etapas finais do processo de moagem, concheamento e refino ocorram de forma mais eficiente.

\section{REFERENCIAS}

CREMASCO, M. A. Operações Unitárias em Sistemas Particulados e Fluidomecânicos. São Paulo: Edgar Blucher, 2012. p. 126-157.

FRARE L. M.; GIMENES, M. L.; PEREIRA N. C; MENDES, E. S. Linearização do modelo log-normal para distribuição de tamanho de partículas. Acta Scientiarum. Technology, v. 22, n. 5, p. 1235-1239, 2000.

MASSARANI, G. Problemas de sistemas particulados. São Paulo: Edgard Blucher, 1984.

OETTERER, M. et al. Tecnologias de obtenção do cacau, produtos do cacau e do chocolate. In: OETTERER, M.; REGITANO-D'ARCE, M. A. B.; SPOTO, M. H. F. Fundamentos de Ciência e Tecnologia de Alimentos. Barueri: Manole, 2006. Cap. 1. p. 1-48.

SCHMITZ, H. H. Chocolate, flavonoids and heart health. Manuf. Confect., Glen Rock, v. 81, n. 9, p. 95-99, 2001.

SCHUMACHER, A.B. Desenvolvimento de um chocolate meio amargo com maior percentual de proteína. Porto Alegre, 2008. 91p. Dissertação (Mestrado) Universidade Federal do Rio Grande do Sul - UFRS. 